\title{
Design, synthesis, and screening of sulfonylurea-derived NLRP3 inflammasome inhibitors
}

\author{
Amol A. Kulkarni ${ }^{1} \cdot$ Ayyiliath M. Sajith ${ }^{1} \cdot$ Trevor T. Duarte $^{2} \cdot$ Anahis Tena $^{2} \cdot$ Charles T. Spencer $^{2} \cdot$ J. Phillip Bowen ${ }^{3}$
}

Received: 19 July 2019 / Accepted: 29 October 2019 / Published online: 14 November 2019

(c) Springer Science+Business Media, LLC, part of Springer Nature 2019

\begin{abstract}
Inflammasomes are multiprotein assemblies that produce robust inflammatory responses upon stimulation with pathogenand/or danger-associated molecular patterns. Uncontrolled inflammasome activation has been linked to the pathophysiology of a wide array of disorders including life-threatening pathogenic infections, e.g., Francisella tularensis. There has been a great deal of interest in the development of small molecule inflammasome inhibitors. Using computational modeling based on chalcone derivatives, we have developed novel tertiary sulfonylurea compounds as inhibitors of the NLRP3 inflammasome. The polar enone functional alert of chalcone was replaced with a sulfonylurea scaffold while maintaining the relative positions of the two aromatic rings. These compounds were evaluated for their ability to inhibit NLRP3 and AIM2 inflammasome activation triggered by Francisella tularensis infection.
\end{abstract}

\section{Introduction}

Inflammasomes are oligomeric multiprotein complexes that function as pattern recognition receptors to trigger the initiation of the inflammasome cascade. The core subunit is typically comprised of procaspase 1 (the ultimate effector protein that initiates inflammation) and apoptosis-associated speck-like protein containing a caspase recruitment domain (ASC), which functions as an adaptor protein between procaspase 1 and a variety of sensor proteins that recognize molecular pattern motifs (Rathinam et al. 2016). Activation of the inflammasome plays a crucial role in a variety of pathophysiological responses when exposed to dangerassociated molecular patterns (DAMPs) and pathogen-

Supplementary information The online version of this article (https:// doi.org/10.1007/s00044-019-02466-7) contains supplementary material, which is available to authorized users.

Amol A. Kulkarni

amol.kulkarni@howard.edu

1 Department of Pharmaceutical Sciences, College of Pharmacy, Howard University, Washington, DC 20059, USA

2 Department of Biological Sciences, University of Texas at El Paso, El Paso, TX 79968, USA

3 Department of Pharmaceutical Sciences, College of Pharmacy, Mercer University, Atlanta, GA 30341, USA associated molecular patterns (PAMPs) (Petrilli et al. 2007). Of particular interest is sensing of DAMPs and PAMPs through the nucleotide binding domain leucine-rich repeat, pyrin-containing 3 (NLRP3) inflammasome. Aberrant activation of the NLRP3 inflammasome is shown to play a crucial role in the pathogenesis of neurodegenerative diseases (e.g., Alzheimer's disease, type-2 diabetes, obesity, multiple sclerosis) and life-threatening pathogenic infections, including the Gram-negative bacterium Francisella tularensis, causative agent of human tularemia (Atianand et al. 2011).

Sensing of DAMPs or PAMPs results in the formation of the multimeric inflammasome complex and autoproteolysis of procaspase 1. Active caspase 1 cleaves interleukin $1 \beta$ (IL-1 $\beta$ ) and interleukin-18 (IL-18) from their precursor forms to the active form (Thornberry et al. ; Martinon et al. 2002; Agostini et al. 2004). The release of active IL-1 $\beta$ and IL-18 subsequently trigger the expression of other inflammatory cytokines, such as IL- 6 , TNF- $\alpha$, IL- 12 , IFN- $\gamma$, etc (Henry and Monack 2007; Mariathasan et al. 2005; Weiss et al. 2007). This response, however, must be tightly controlled as its dysregulation can result in the pathological cytokine storm (Tisoncik et al. 2012). In the case of $F$. tularensis infection, excessive pro-inflammatory cytokine production has been shown to progress into edema, hemorrhage, hypovolemic shock, acute respiratory distress syndrome, and, if left untreated, death (Mares et al. 2008; Sharma et al. 2011). Mice deficient in inflammasome 
activation are more resistant to $F$. tularensis infection (Mariathasan et al. 2005; Periasamy et al. 2016). Moreover, mice deficient in both IL-1 $\beta$ and IL-18, products of inflammasome activation, are more susceptible to $F$. tularensis infection compared with the wild type mice. Conversely, mice deficient in only one of these products are protected (Collazo et al. 2006). Therefore, suppression but not ablation of inflammasome function seems to be a promising approach for preventing the overactivation of the inflammatory cytokine storm during $F$. tularensis infection.

Given the central role of the NLRP3 inflammasome in this and other pathological and pathophysiological processes, there is a profound interest in the development of small molecules with NLRP3 inhibitory activity. Glyburide, a drug used in the treatment of type- 2 diabetes was shown to display NLRP3 inhibitory activity at high millimolar concentrations (Lamkanfi et al. 2009). In addition, MCC950, a sulfonylurea-based compound, showed promising activity in animal models of multiple sclerosis (Coll et al. 2015). Polyphenolic compounds such as curcumin (Yin et al. 2018), resveratrol (Chang et al. 2015), isoliquiritigenin (Honda et al. 2014), have been identified as NLRP3 inflammasome inhibitors. Small molecules that form covalent interactions with NLRP3 inflammasome are also reported. However, the structure of neither the NLRP3 inflammasome nor its individual component proteins has been determined. This lack of structural details has proven to be a major impediment in the rational design of small molecule inhibitors capable of specifically inhibiting the uncontrolled inflammasome activation. In this article, we report the synthesis of rationally-designed small molecule NLRP3 inflammasome inhibitors using computational chemistry and pharmacophore modeling. The cyclic, tertiary sulfonylurea compounds were geometry optimized at the Hartree-Fock level of theory using the 6-31 G(d) basis set using Spartan '16 molecular modeling software (Pro 2018). The pharmacophoric regions were aligned using curcumin derivatives as the lead molecule. These novel rationally-designed inflammasome inhibitors were screened in vitro against Francisella tularensis-induced NLRP3 inflammasome activation.

\section{Results and discussion}

\section{Molecular modeling and chemistry}

Curcumin, a bis-phenolic natural product isolated from the rhizomes of Curcuma longa, has been reported to have diverse and promising pharmacological activity that could be used to treat a wide array of disease states. The fact that curcumin has a number of biological effects has labeled curcumin as a Pan-assay interference compound (PAINS), but this does not preclude this natural product as a scaffold for drug development (Capuzzi et al. 2017). Most recently, curcumin was shown to bind to dual-specificity tyrosineregulated kinase 2 (Banerjee et al. 2018). Also, curcumin and the chalcone derivative isoliquiritigenin are known inhibitors of the NLRP3 inflammasome. Curcumin, however, has a low plasma half-life because of its facile degradation via a retro-aldol pathway (Anand et al. 2007). It also metabolized into pharmacologically inactive glucuronide metabolite. Thus, chemical and enzymatic instability of this natural product, present some serious challenges from the drug discovery perspective.

Previous studies have shown that removal of the $\beta$-diketone moiety significantly improves the chemical stability, but there still remain serious pharmacokinetic concerns (Robinson et al. 2003, 2005). The curcuminoids and chalcones are two promising structural scaffolds that can be used for the development of potential inflammasome inhibitors (Fig. 1). Nevertheless, aromatic hydroxyl derivatives of the curcuminoids and chalcones are still vulnerable to facile phenolic glucuronidation. Moreover, the $\alpha$, $\beta$-unsaturated carbonyl moiety can undergo potential and problematic Michael additions with glutathione. We envisioned using chalcone and curcuminoid scaffolds as our starting point for the development of new series of potential inflammasome inhibitors using the following stratagem.

Curcumin can be divided into three critical regions (Fig. 2). Both regions A and C are comprised of substituted aromatic rings, which we hypothesized, played a critical role in binding. The tolerance in the distance between the centroids of these two rings and their orientation have not been explored fully. Region B (red) is comprised of an electrophilic $\alpha, \beta$-unsaturated carbonyl system, shown below in an enol form. Our goal was to modify this electrophilic linker, which connects the two aromatic regions, to eliminate the $\alpha, \beta$-unsaturated carbonyl system.

The electrophilic enone linker region can undergo Michael (1,4-addition) reactions with various nucleophiles to form covalent bonds. While there are drugs with the enone functional group, it is considered a structural alert because of the potential for depleting glutathione concentrations. Previously we (Kumari et al. 2015) and others (Deck et al. 2018) have shown that the linker region can be modified. Figure 3 shows that a similar division of the enone and dienone into three regions.

We used the two aromatic pharmacophoric centers and the hydrogen-bond acceptor pharmacophoric center of the carbonyl of chalcone as our starting point. Figure 4 shows the alignment of curcumin and chalcone. We hypothesized that the linker (region B) could be replaced with a sulfonylurea. One scaffold that connects the two aromatic ring systems with a six-membered sulfonylurea is shown in Fig. 5. The sulfonyl group both in terms of electrostatics 
Fig. 1 The structure of curcumin in the keto-enol form is shown. This natural product has served as the lead structure for the development of two different classes of derivatives: the chalcones and the mono-ketone curcuminoids

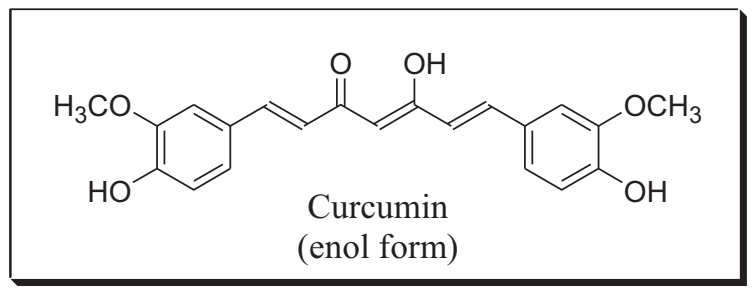

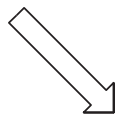<smiles>[R][R1]1ccc(/C=C/C(=O)c2cccc[R]2[R])cc1</smiles>

Chalcones<smiles>[R][R]1cccc(/C=C/C(=O)/C=C/c2ccc(O)c[R]2[H])c1</smiles>

Curcuminoids

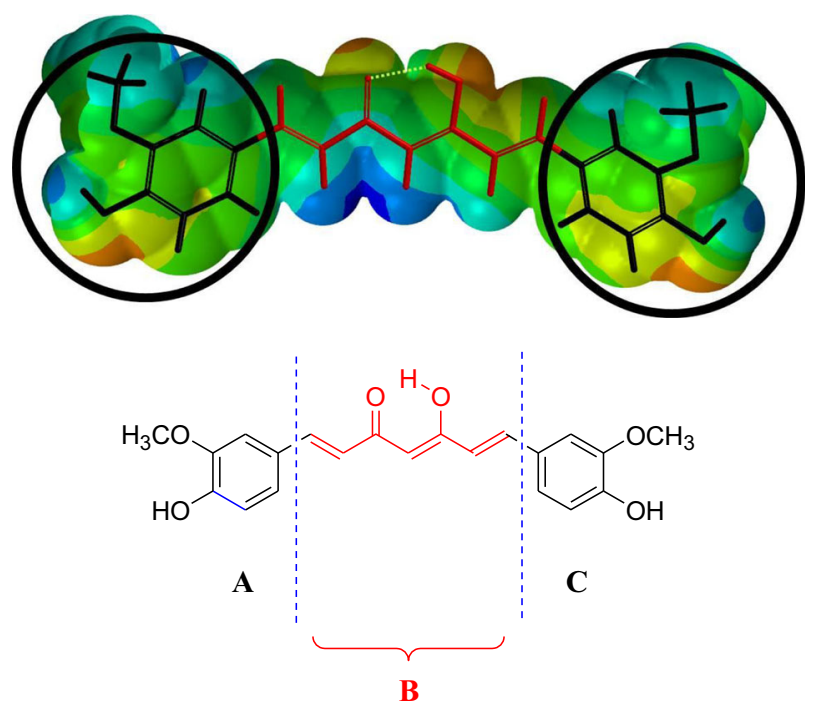

Fig. 2 The lead structure of curcumin is divided into three regions. Both regions $\mathrm{A}$ and $\mathrm{C}$, with the molecular skeleton shown in black, are aromatic rings which are linked via an electrophilic unsaturated carbonyl system, region B. A line drawing of curcumin is displayed at the bottom of the figure. The natural product was energy minimized in Spartan at the Hartree-Fock level of theory using the 6-31 G(d) basis set, and it is enveloped in its electrostatic potential energy surface. The electrostatic potential energy surface is color coded, where red depicts a more electronegative region and blue depicts a more electropositive region

and length was a reasonable mimic for the region $\mathrm{B}$ of chalcone and the enol form of curcumin. Our computational studies described below indicate that diaromatic sulfonylurea structures can adopt conformations that place the aromatic rings in similar positions in 3D space. Our goal was not to necessarily have identical placement of the aromatic ring systems because we were interested in exploring what tolerance of the putative binding site and to develop novel lead compounds.

Chalcone and a series of diaromatic sulfonylurea structures were energy minimized at the Hartree-Fock level of theory using the 6-31 G(d) basis set. Frequency calculations were carried out to verify that minimum energy structures were achieved. While we do not necessarily expect the bioactive conformations to be identical with energy minima, we decided to use the latter as a first approximation. Using the pharmacophoric pattern of chalcone as our model, we were able to align the six-membered and five-membered sulfonylurea molecular structures with an excellent fit (Fig. 6). Note the proximity of the pharmacophoric carbonyl oxygen electron density with the sulfonyl oxygen electron density. The pharmacophore model has the two aromatic rings of the sulfonylureas occupying essentially the same 3D space as chalcone. Presumably there is enough conformational flexibility of the aromatic rings to provide an even better structural fit, but energy minimized structures are shown in Fig. 6. The sulfonyl oxygen atoms occupy similar conformational space as the carbonyl oxygen of chalcone.

\section{Chemical synthesis}

Chemical synthesis of the tertiary sulfonylurea structures is outlined in Scheme 1. It involves a reaction of 4substituted aniline derivative with the appropriate chloroalkyl isocyanate resulting in the formation of acyclic urea 2 and 3. Base-mediated cyclization of the latter followed by reaction with the appropriate arylsulfonyl chloride resulted in the isolated of the desired $3^{\circ}$-sulfonylurea products $4-7$. 
Fig. 3 The chalcone and dienone derivatives of curcumin are shown in the same simple twodimensional outline. From a drug design perspective, the goal is to find a linker that connects the two aromatic rings<smiles>COc1cc(I)cc(C(=O)C=Cc2cc(O)c(O)cc2I)c1</smiles>

B<smiles>COc1cc(I)cc(C=CC(=O)C=Cc2cc(O)c(O)cc2I)c1</smiles>

B
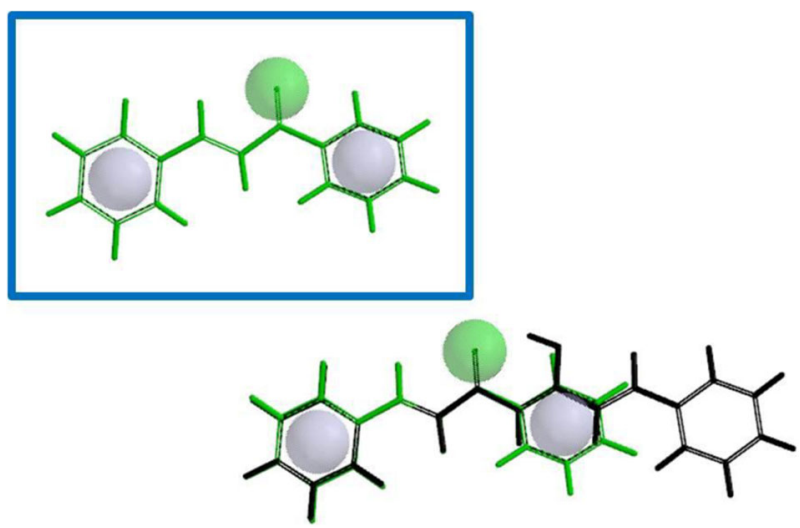

Fig. 4 Chalcone (green) and the enol form of curcumin (black) were energy minimized at the Hartree-Fock level of theory using the 6-31 G(d) basis set. Curcumin and chalcone were aligned in Spartan using the pharmacophoric groups of the latter

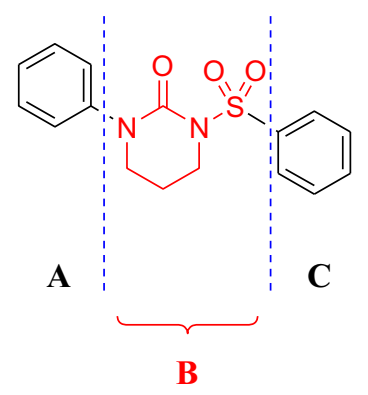

Fig. 5 The sulfyonylureas system can be used to link the two aromatic regions $\mathrm{A}$ and $\mathrm{C}$

\subsection{Biological screening}

In order to prevent direct killing of the mammalian host cells, cytotoxicity of the compounds was determined against uninfected and infected mammalian macrophage cell lines. Uninfected cells were cultured with titrating amounts of inflammasome inhibitor for $48 \mathrm{~h}$ and then, stained with propidium iodide to label dead and dying cells. These cultures were imaged on a BD Pathway Confocal imager and quantified by confocal fluorescence counting. Inflammasome inhibitor compounds 4,5 , and 6 were mildly cytotoxic at the highest concentration tested $(1 \mathrm{mM})$ while compound 7 was highly cytotoxic at the highest concentration

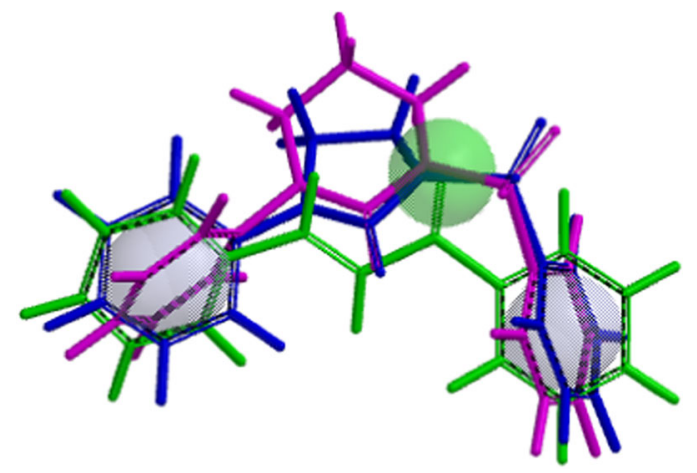

Fig. 6 The scaffolds of chalcone (green), the five-membered sulfonylurea (blue), and the six-membered sulfonylurea (purple) are shown. In this overlay, all of the structures have been aligned in Spartan based on the chalcone pharmacophore model using energy minimized structures at the Hartree-Fock level of theory with the 6-31 G(d) basis set

(Fig. 7a). Infection of macrophages by $F$. tularensis is known to induce some cell death (d.n.s); however, addition of $100 \mu \mathrm{M}$ of the inhibitor compounds did not induce additional cell death as measured by PI staining (Fig. 7b). To prevent misinterpretation of data, all subsequent experiments were performed at inflammasome inhibitor compound concentrations at or below $100 \mu \mathrm{M}$.

Macrophage sensing of $F$. tularensis infection differently relies on the NLRP3- or AIM2-dependent inflammasome in human and mouse cells, respectively. These rationallydesigned $3^{\circ}$-sulfonylurea compounds were designed to prevent inflammation by disrupting formation of the NLRP3 inflammasome. Initiation of inflammation involves an orderly stepwise process resulting in proteolytic processing and release of $\mathrm{IL}-1 \beta$ and subsequent signaling to induce secretion of inflammatory cytokines, e.g., IL-6. Functional activation of the inflammasome results in accumulation of these cytokines in the supernatant providing a rapid measure of the effects of the inhibitors. Pre-treatment of macrophages with inflammasome inhibitor compounds prior to infection did not alter production of either IL-1 $\beta$ or IL-6 in response to infection with $F$. tularensis with the inhibitor being removed prior to infection (Supplementary Fig. 1). 


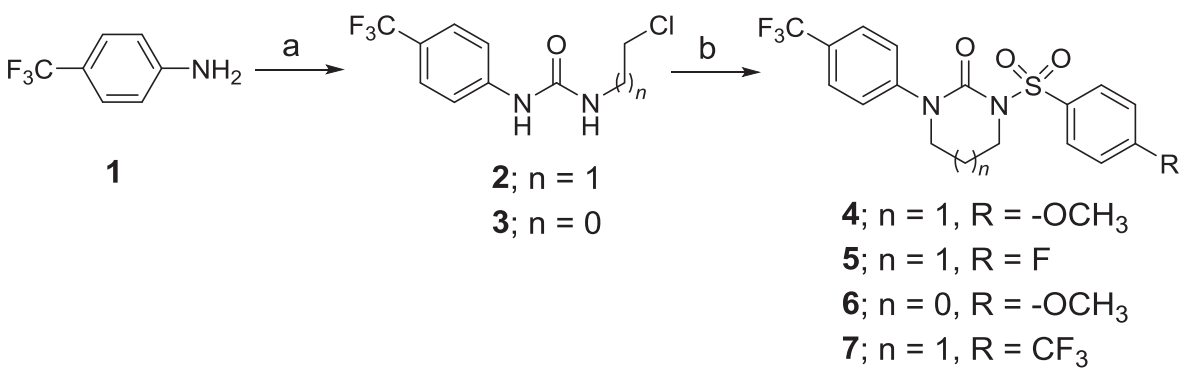

Scheme 1 Synthesis of tertiary sulfonylureas a 2chloroethylisocyanate $(n=0)$ or 3-chloropropylisocyanate $(n=1)$, $\mathrm{CH}_{2} \mathrm{Cl}_{2}, \mathrm{rt},(87-89 \%) ;$ b 1. NaH (1 equivalent), 2. 4-methoxybenzene sulfonyl chloride, 4-fluorobenzene sulfonyl chloride or 4trifluromethylbenzene sulfonyl chloride ( $80-88 \%$ overall yield over two steps)
A

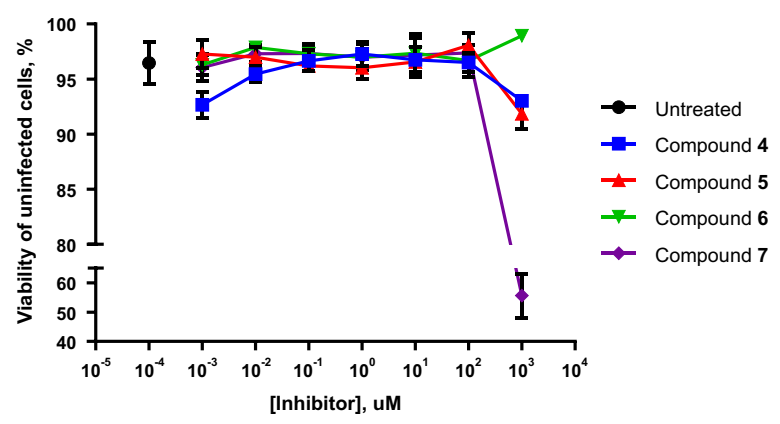

B

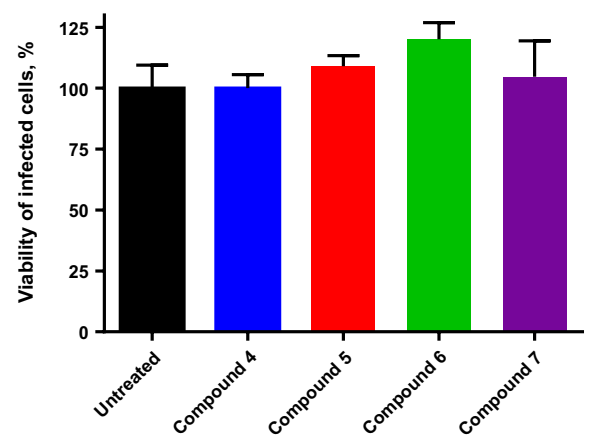

Fig. 7 Toxicity of compounds 4-7. a Propidium iodide staining of uninfected cells analyzed on a BD Pathway Bioimager. Untreated cell viability is represented by the black circle. No differences in the total cell number were observed. b Propidium iodide staining of cells

Addition of $100 \mu \mathrm{M}$ inflammasome inhibitors during infection of mouse or human macrophages with $F$. tularensis, however, did result in inhibition of inflammation as measured by the accumulation of IL- $1 \beta$ and IL- 6 in the supernatant after $48 \mathrm{~h}$. Compounds $\mathbf{4 , 5}$, and $\mathbf{7}$ effectively inhibited IL-1 $\beta$ production, $>80 \%$ suppression, from infected mouse macrophages (Fig. 8a) while only compound 4 efficiently suppressed downstream IL-6 secretion (Fig. 8b). Compound 6 was completely ineffective at suppressing cytokine production in mouse cells. In infected human cells, compounds 4,6 , and 7 mildly inhibited IL- $1 \beta$ production, 15-20\% suppression (Fig. 8c), while compound 7 was most effective at inhibiting downstream IL-6 production (Fig. 8d). These data suggested that while compound 4 strongly inhibits inflammasome activation, it is likely specific to the AIM2-mediated inflammasome rather than NLRP3. In contrast, compound $\mathbf{7}$ has a strong specificity for the NLRP3-mediated inflammasome.

To verify that these compounds disrupt formation of the inflammasome, treated macrophages were stained with antibodies against the ASC and either AIM2 (Fig. 9a) or NLRP3 (Fig. 9b) subunits of the inflammasome. These cells infected with $F$. tularensis treated with $100 \mu \mathrm{M}$ compounds 4-7 analyzed on a BD Pathway Bioimager compared with untreated infected cells. Data is representative of three independent experiments

were imaged by confocal microscopy to determine the extent of association between ASC (red) and the NLRP3 or AIM2 subunits (green). Resting cells segregate the fluorescent signals and appear either red or green (Fig. 9a, Uninfected) while $F$. tularensis infection induces formation of the inflammasome observed as the overlap of the fluorescent signal resulting in yellow cells (Fig. 9a, Infected) which was unaltered by vehicle treatment (Fig. 9a, Vehicle). Addition of Compound $\mathbf{4}$ to murine cells strongly prevented overlap of the AIM2-ASC signal. This effect titrated out at $1 \mu \mathrm{M}$. In contrast, compounds 5 and $\mathbf{7}$ were less effective at preventing overlap of the fluorescent signal. Compound $\mathbf{6}$ did not prevent overlap of the inflammasome subunit at any concentration tested. The previously reported inhibitor, glyburide, was also ineffective at preventing overlap of the fluorescent signal at the concentrations tested here while MCC950 treatment did segregate the inflammasome subunits. Similarly, infection of human macrophages triggered global inflammasome formation (Fig. 9b, Infected). This was effectively blocked by compounds $\mathbf{4}, \mathbf{5}$ and $\mathbf{7}$ but was unaffected by compound 6 (Fig. 9b). Of these, compound 7 was most effective at preventing inflammasome formation. 
A
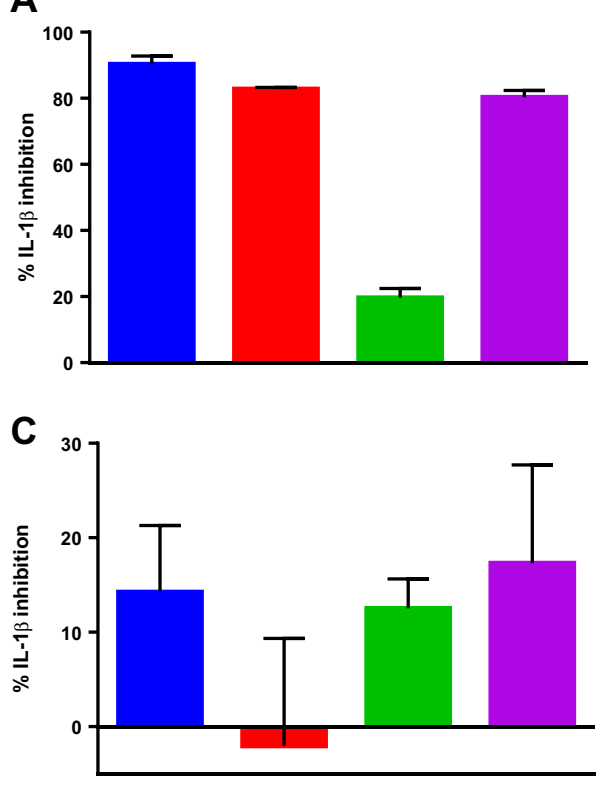

B

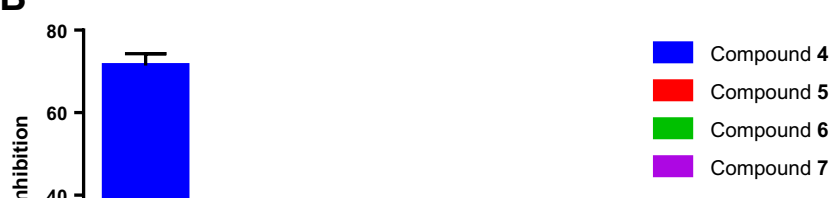

Fig. 8 Inhibition of inflammation elicited by $F$. tularensis infection of mouse (a, b) and human (c, d) macrophages. Infected macrophages were treated with $100 \mu \mathrm{M}$ inhibitor for $48 \mathrm{~h}$ and production of IL-1 $\beta$ $(\mathbf{a}, \mathbf{c})$ and IL-6 (b, d) was quantified by ELISA. Representative data is presented from three replicate experiments
Glyburide was more effective at blocking inflammasome formation in human cells than mouse cells while MCC950 continued to be effective.

\section{Conclusion}

Herein, we describe a strategy for the identification of curcumin-inspired small molecule NLRP3 inflammasome inhibitors based on pharmacophoric and structural alignments using geometry optimized structures with the Hartree-Fock level of theory using the 6-31 G(d) basis set. Unique features of our pharmacophore modeling studies include the substitution of the sulfonylurea moiety for the enone linker present in curcumin and chalcone, while preserving the two regions with electron rich aromatic rings. Our synthetic protocol is efficient and compatible with a wide-array of commonly utilized functional groups.

In addition, this study establishes a general in vitro assay system for the screening of NLRP3 inhibitors. Despite the production of IL-1 $\beta$ as the direct result of inflammasome activation, we found that the downstream production of IL6 in response to concomitant inflammasome activation and TLR-mediated signaling to be a more sensitive measure of the inhibition of inflammation. Because IL- $1 \beta$ is the originating signal priming immune cells to respond by inducing inflammation, small changes in IL-1 $\beta$ can result in large changes in inflammation. As a result of these small changes in IL-1 $\beta$, it is difficult to observe differences between potential inhibitors. Since the production of IL-6 results from an amplification of the inflammatory signal, these differences are augmented and much more easily observed. It is critical, however, to confirm that IL- $1 \beta$ production is directly suppressed by these inhibitors since we observed that compound 5 inhibited IL- 6 production but not IL-1 $\beta$ production in human cells. This suggests that compound $\mathbf{5}$ may actually inhibit TLR-mediated signaling rather than the inflammasome signaling. Further experimentation along these lines may identify the proteins directly suppressed by compound 5. Surprisingly, IL-1 $\beta$ production was consistently inhibited more strongly in murine macrophages compared with human macrophages. Though unexplained, this may suggest a differential reliance on IL-1 $\beta$-mediated triggering of inflammation or an as yet incomplete picture of the NLRP3 inflammasome structure.

Cumulatively, our results demonstrate that compound 4 displayed greater inhibitory activity in murine cells, which rely more on AIM2 to sense bacterial infection and activate the inflammasome. Compound $\mathbf{7}$ displayed greater inhibitor activity in human cells which rely more on NLRP3 to sense bacterial infection and activate the inflammasome (Atianand et al. 2011). Compound $\mathbf{6}$ was completely ineffective at inhibiting inflammasome activation. While compound 5 inhibited the production of IL6 it did not inhibit IL-1 $\beta$. These results were consistent in analysis of the secretion of cytokines following infection and the overlap of fluorescent signals in formation of the inflammasome complex. 
A
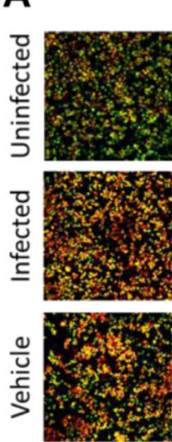

B
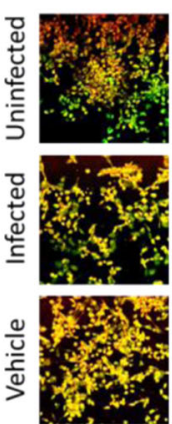
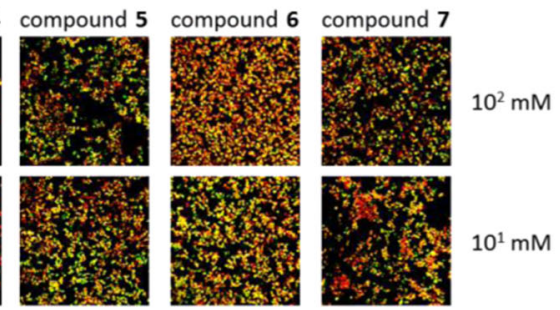

$10^{1} \mathrm{mM}$

Glyburide

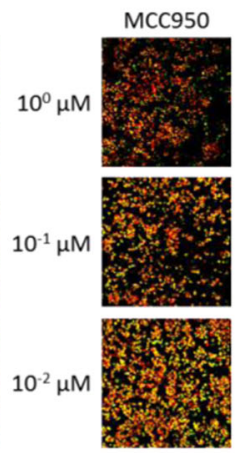

$10^{\circ} \mu \mathrm{M}$
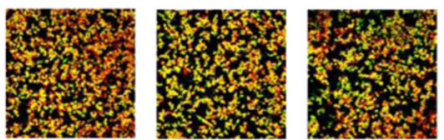

$10^{\circ} \mathrm{mM}$
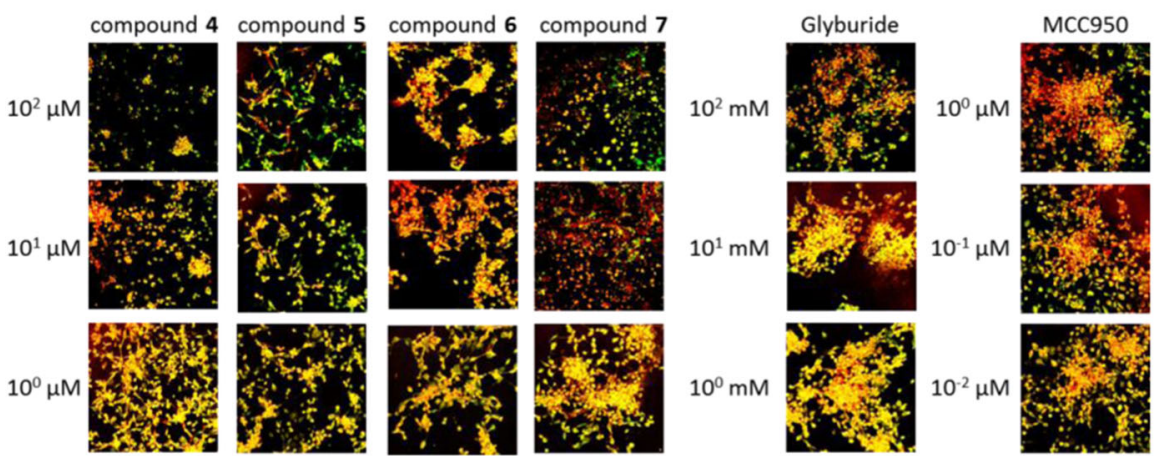

Fig. 9 Inhibition of inflammasome formation. Infected murine a or human $\mathbf{b}$ macrophages were treated with indicated concentrations of inhibitors and stained for AIM2 (A, green) or NLRP3 (b, green) and ASC $(\mathbf{a} / \mathbf{b}$, red). Uninfected cells displayed little overlap in fluorescent signal with red and green distinctly separated whereas infected cells

displayed overlapped fluorescent signal (yellow) indicating formation of the inflammasome. Addition of effective inhibitors resulted in separation of the fluorescent signal. Representative figures from two replicate experiments

\section{Experimental section}

\section{General}

Glassware for chemical synthesis was dried in an oven $\left(120^{\circ} \mathrm{C}\right)$ and was cooled under argon before use. Unless otherwise noted, materials obtained from the commercial suppliers were used without any purification. Reactions were monitored via thin-layer chromatography on Analtech silica gel plates and visualized under UV-light. Column chromatography was performed on silica gel (23-400 mesh) purchased from Sigma-Aldrich. Evaporation of the volatile components was conducted under reduced pressure using a rotary evaporator. Fourier Transform-Infrared (FT-IR) spectra were recorded neat on a PerkinElmer Spectrum 65 instrument. Nuclear magnetic resonance (NMR) spectra were recorded on a Bruker Avance III 400 NME spectrometer at $400 \mathrm{MHz}\left({ }^{1} \mathrm{H}\right)$ and $100 \mathrm{MHz}\left({ }^{13} \mathrm{C}\right)$ respectively in deuterated chloroform $\left(\mathrm{CDCl}_{3}\right)$ solvent. The spectra were calibrated against residual solvent peaks $\left(7.24 \mathrm{ppm}\right.$ for ${ }^{1} \mathrm{H}$ and $77.0 \mathrm{ppm}$ for $\left.{ }^{13} \mathrm{C}\right)$. Chemical shifts $(\delta)$ and coupling constants $(J)$ are reported in parts per million (ppm) and Hertz $(\mathrm{Hz})$ respectively. The following abbreviations were used to explain multiplicities: $\mathrm{s}=$ singlet, $\mathrm{d}=$ doublet,

$\mathrm{t}=$ triplet, $\mathrm{q}=$ quartet, $\mathrm{m}=$ multiplet, $\mathrm{bs}=$ broad singlet. Low resolution ESI mass spectra were obtained on a Waters Acquity UPLC H-Class with PDA and SQ mass detectors using a Waters BEH C18 $1.7 \mathrm{~lm}$ column $(2.150 \mathrm{~mm})$. High resolution mass spectra were obtained on VG $70-70 \mathrm{H}$ or LC/MSD trapSL spectrometer operating at $70 \mathrm{eV}$ using a direct inlet system.

\section{Synthetic procedures}

Chemical synthesis of compounds 2-6 has been described previously (Xu et al. 2018).

1-(4-(trifluoromethyl)phenyl)-3-((4-(trifluoromethyl)phenyl)sulfonyl)tetrahydropyrimidin-2(1H)-one (7): A round bottom flask ( $10 \mathrm{~mL}$ capacity) equipped with a magnetic stir bar was charged with a solution of acyclic urea $(70 \mathrm{mg}$, 0.25 mmole, 1.0 equiv) in THF $(2 \mathrm{~mL})$. The solution was cooled to $0{ }^{\circ} \mathrm{C}$ for $15 \mathrm{~min}$. $\mathrm{NaH}(30 \mathrm{mg}, 0.75 \mathrm{mmole}$, 3.0 equiv, $60 \%$ suspension in oil) was slowly added. The reaction was stirred for $15 \mathrm{mins}$ at $0{ }^{\circ} \mathrm{C}$ for $30 \mathrm{~min}$, slowly brought to RT, and stirred at RT for $12 \mathrm{~h}$. After $12 \mathrm{~h}$, 4-trifluoromethylbenzenesulfonyl chloride (68 mg, 0.28 mmole, 1.1 equiv) was added and the reaction was stirred at RT for additional $4 \mathrm{~h}$. The reaction mixture was 
poured into separatory funnel containing ethyl acetate and $1 \mathrm{M} \mathrm{HCl}$ (10 mL each). The layers were separated, organic layer was washed with distilled $\mathrm{H}_{2} \mathrm{O}(2 \times 10 \mathrm{~mL})$, and dried using anhydrous $\mathrm{Na}_{2} \mathrm{SO}_{4}$. Solvent was removed using a rotary evaporator. The crude product was purified using silica gel flash column chromatography as a white solid.

7. ${ }^{1} \mathrm{H} \mathrm{NMR}\left(400 \mathrm{MHz}, \mathrm{CDCl}_{3}\right) \delta 8.15-8.13(\mathrm{~d}, 2 \mathrm{H})$, $7.76-7.74(\mathrm{~d}, J=12.6 \mathrm{~Hz}, 2 \mathrm{H}), 7.60-7.57(\mathrm{~d}, J=8.3 \mathrm{~Hz}$, $2 \mathrm{H}), 7.35-7.33(\mathrm{~m}, 2 \mathrm{H}), 4.14-4.11(\mathrm{t}, 2 \mathrm{H}), 3.74-3.71$ $(\mathrm{t}, 2 \mathrm{H}), 2.31-2.25(\mathrm{~m}, 2 \mathrm{H}) .{ }^{13} \mathrm{C} \mathrm{NMR}\left(100 \mathrm{MHz}, \mathrm{CDCl}_{3}\right)$ : $149.98,144.14,142.42,134.41,128.47,125.67,125.64$, $125.60,125.56,125.38,125.26,125.22,125.18,125.42$, $123.87,121.72,121.16,48.43,44.90,22.19$. IR $\mathrm{cm}^{-1}$ : 2917.3, 2854.6, 1673.8, 1611, 1473.8, 1426.7, 1313, 1163.9, 1061.9, 1101.1, 842.21, 716.69. HRMS (ESI) $\mathrm{m} / \mathrm{z}$ $453.0706[\mathrm{~m}+\mathrm{H}]^{+}$.

\section{Biological evaluation}

\section{Cell culture}

THP-1 monocytes (ATCC TIB-202, Manassas, VA) were maintained in RPMI-1640 containing $2 \mathrm{mM}$ glutamine, $2 \mathrm{mM}$ glucose supplemented with $10 \%$ FBS and $10 \mathrm{mM} 2-$ mercaptoethanol. For differentiation, THP-1 cells were plated in 96 well plates at $1 \times 10^{5}$ cells per well and incubated for $24 \mathrm{~h}\left(37^{\circ} \mathrm{C}, 5 \% \mathrm{CO}_{2}\right)$ in the presence of $100 \mathrm{nM}$ phorbol myristate acetate (PMA). Following differentiation, cells were allowed to recover for $24 \mathrm{~h}$ in fresh culture media prior to use in subsequent experiments. J virus immortalized bone marrow derived murine macrophages (BEI Resources NR-9456, Manassas, VA) were maintained in DMEM containing $2 \mathrm{mM}$ glutamine, $2 \mathrm{mM}$ glucose supplemented with $10 \%$ FBS, $1 \%$ non-essential amino acids, $1 \%$ sodium pyruvate and $1 \%$ HEPES.

\section{Compound preparation}

All compounds were dissolved in DMSO and stored at $-20{ }^{\circ} \mathrm{C}$ until use. Working stocks $(10 \mathrm{mM})$ were diluted in DMSO, placed in amber borosilicate glass tubes and stored at $4{ }^{\circ} \mathrm{C}$.

\section{Toxicity assay}

Murine macrophages or PMA-differentiated THP-1 cells were plated in flat bottomed 96 well plates at a cell density of $1 \times 10^{4}$ cells per well. Cells were incubated with compounds A-D ( $1 \mathrm{mM}$ to $10 \mathrm{nM}$, in six 10-fold dilutions) for $24 \mathrm{~h}$. After incubation macrophages were stained with propidium iodide and Hoechst 33342 ( $1 \mu \mathrm{g} / \mathrm{ml}$ final concentration) and analyzed for viability on a BD pathway 855 bioimager as described previously (Lema et al. 2011).

\section{Activation and inhibition of inflammasome}

Murine macrophages or PMA-differentiated THP-1 cells were plated to a density of $1 \times 10^{5}$ cells per well in flat bottomed 96 well plates. Cells were infected (MOI 40) for $2 \mathrm{~h}$ with $F$. tularensis live vaccine strain (LVS). Following infection, cells were washed twice with PBS pH 7.2, cultured in fresh culture media containing $50 \mu \mathrm{g} / \mathrm{mL}$ gentamycin and incubated for an additional 16-24h. For compound testing, cells were incubated with $1 \mathrm{mM}$ to $1 \mathrm{nM}$ final concentration of test compound for $2 \mathrm{~h}$ pre-infection or for $24 \mathrm{~h}$ post-infection in RPMI media containing $50 \mu \mathrm{g} / \mathrm{mL}$ gentamycin. The following day conditioned media was collected and stored at $-20^{\circ} \mathrm{C}$ for cytokine analysis. Proinflammatory cytokines (IL-1 $\beta$ and IL-6) were independently measured by sandwich ELISA following manufacturer's protocol (BD Bioscience, San Jose, CA). For each experiment, $50 \mu \mathrm{L}$ of undiluted conditioned media was assayed.

\section{Microscopic analysis of inflammasome formation and activation}

Murine macrophages or PMA-differentiated THP-1 cells were plated in flat bottomed 96 well plates at a cell density of $1 \times 10^{5}$ cells per well. Cells were incubated with compounds A-D $(100 \mu \mathrm{M}$ to $1 \mathrm{nM}$, in seven ten-fold dilutions) for $24 \mathrm{~h}$. Cells were infected (MOI 40) for $2 \mathrm{~h}$ with $F$. tularensis live vaccine strain (LVS). Following infection, cells were washed twice with PBS $\mathrm{pH} 7.2$, cultured in fresh culture media containing $50 \mu \mathrm{g} / \mathrm{mL}$ gentamycin and incubated for an additional 16-24 h. Macrophages were fixed for $15 \mathrm{~min}$ at room temperature with $4 \%$ formaldehyde in PBS and then were washed three times with PBS. Nonspecific binding in cells was blocked with $1 \%$ bovine serum albumin and cells were permeabilized with $0.1 \%$ Triton X-100 for 5 min. Cells were blocked with $1 \%$ bovine serum albumin in PBST for 30 minutes and then incubated with anti-ASC (Santa Cruz Biotechnology), anti-NLRP3 and anti-ASC antibodies (Abcam) for $1 \mathrm{~h}$ (Ke et al. 2017; Granata et al. 2015; Baroja-Mazo et al. 2014) and imaged on Zeiss LSM700 at $\times 20$ or $\times 63$ magnification.

\section{Computational}

All the calculations were carried out in the Spartan molecular modeling program in the ground state in the gas phase. Chalcone and the diaromatic sulfonylurea structures were energy minimized using the Equilibrium Geometry option in Spartan at the Hartree-Fock level of theory using the 6-31 G(d) basis set and with molecular mechanics (MMFF). For each geometry optimization, a frequency 
calculation was used to verify that an energy minimum structure was located successfully.

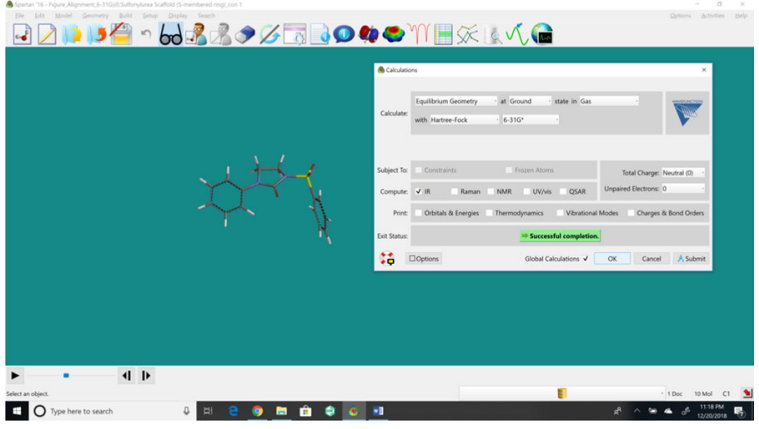

The final stable conformers for the sulfonylurea structures were achieved manually by initially generating the $+60,-60$, and 180 conformations for the flexible dihedral angles followed by energy minimization as described above. Additionally, rotational profiles were generated to verify the results.

While there are multiple alignments possible, the structures were aligned using the Geometry option in Spartan is based on the chalcone pharmacophore model described in the paper. The energy minimized structures achieved with Hartree-Fock level of theory with the 6-31 G(d) basis set and MMFF were used. Alignments based on either the molecular structures or the aromatic ring pharmacophoric centers gave similar results.

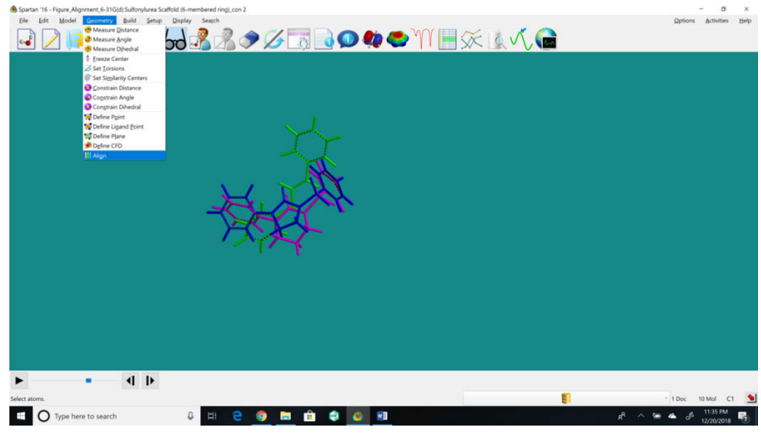

Electrostatic potential maps were generated under the Display option in Spartan.

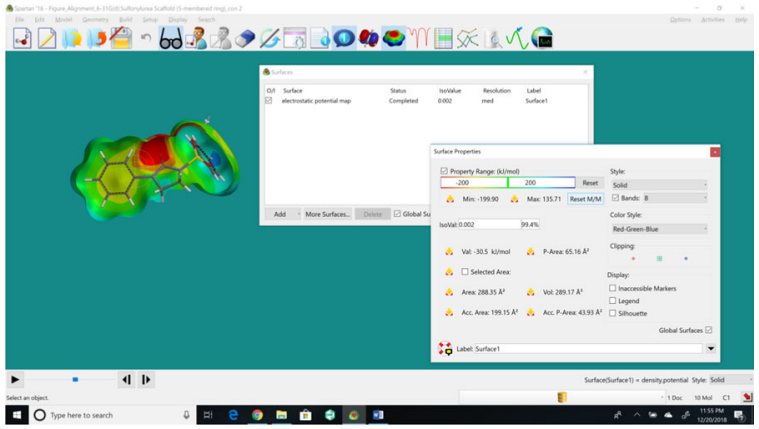

Acknowledgements This work was supported in part by the District of Columbia Center for AIDS Research (DC CFAR; AI117970) and Research Centers in Minority Institutions (RCMI), Howard University. Biological research reported in this article was supported by the National Institute of General Medical Sciences of the National Institutes of Health under linked Award Numbers RL5GM118969, TL4GM118971 and UL1GM118970. The content is solely the responsibility of the authors and does not necessarily represent the official views of the National Institutes of Health

\section{Compliance with ethical standards}

Conflict of interest The authors declare that they have no conflict of interest.

Publisher's note Springer Nature remains neutral with regard to jurisdictional claims in published maps and institutional affiliations.

\section{References}

Agostini L, Martinon F, Burns K, McDermott MF, Hawkins PN, Tschopp J (2004) NALP3 forms an IL-1 $\beta$-processing inflammasome with increased activity in muckle-wells autoinflammatory disorder. Immunity 20(3):319-325

Anand P, Kunnumakkara AB, Newman RA, Aggarwal BB (2007) Bioavailability of curcumin: problems and promises. Mol Pharm 4(6):807-818

Atianand MK, Duffy EB, Shah A, Kar S, Malik M, Harton JA (2011) Francisella tularensis reveals a disparity between human and mouse NLRP3 inflammasome activation. J Biol Chem 286 (45):39033-39042

Banerjee S, Ji C, Mayfield JE, Goel A, Xiao J, Dixon JE, Guo X (2018) Ancient drug curcumin impedes 26S proteasome activity by direct inhibition of dual-specificity tyrosine-regulated kinase 2. Proc Natl Acad Sci U S A 115(32):8155-8160

Baroja-Mazo A, Martin-Sanchez F, Gomez AI, Martinez CM, Amores-Iniesta J, Compan V, Barbera-Cremades M, Yague J, Ruiz-Ortiz E, Anton J, Bujan S, Couillin I, Brough D, Arostegui JI, Pelegrin P (2014) The NLRP3 inflammasome is released as a particulate danger signal that amplifies the inflammatory response. Nat Immunol 15(8):738-748

Capuzzi SJ, Muratov EN, Tropsha A (2017) Phantom PAINS: problems with the utility of alerts for pan-assay interference compounds. J Chem Inform Model 57(3):417-427

Chang YP, Ka SM, Hsu WH, Chen A, Chao LK, Lin CC, Hsieh CC, Chen MC, Chiu HW, Ho CL (2015) Resveratrol inhibits NLRP3 inflammasome activation by preserving mitochondrial integrity and augmenting autophagy. J Cell Physiol 230(7):1567-1579

Coll RC, Robertson AAB, Chae JJ, Higgins SC, Munoz-Planillo R, Inserra MC, Vetter I, Dungan LS, Monks BG, Stutz A, Croker DE, Butler MS, Haneklaus M, Sutton CE, Nunez G, Latz E, Kastner DL, Mills KHG, Masters SL, Schroder K, Cooper MA, O'Neill LAJ (2015) A small-molecule inhibitor of the NLRP3 inflammasome for the treatment of inflammatory diseases. Nat Med 21(3):248-255

Collazo CM, Sher A, Meierovics AI, Elkins KL (2006) Myeloid differentiation factor-88 (MyD88) is essential for control of primary in vivo Francisella tularensis LVS infection, but not for control of intra-macrophage bacterial replication. Microbes Infect 8 (3):779-790

Deck LM, Hunsaker LA, Vander Jagt TA, Whalen LJ, Royer RE, Vander Jagt DL (2018) Activation of anti-oxidant Nrf2 signaling by enone analogues of curcumin. Eur J Med Chem 143:854-865 
Granata S, Masola V, Zoratti E, Scupoli MT, Baruzzi A, Messa M, Sallustio F, Gesualdo L, Lupo A, Zaza G (2015) NLRP3 inflammasome activation in dialyzed chronic kidney disease patients. PLoS ONE 10(3):e0122272

Henry T, Monack DM (2007) Activation of the inflammasome upon Francisella tularensis infection: interplay of innate immune pathways and virulence factors. Cell Microbiol 9(11):2543-2551

Honda $\mathrm{H}$, Nagai $\mathrm{Y}$, Matsunaga $\mathrm{T}$, Okamoto N, Watanabe Y, Tsuneyama K, Hayashi H, Fujii I, Ikutani M, Hirai Y, Muraguchi A, Takatsu K (2014) Isoliquiritigenin is a potent inhibitor of NLRP3 inflammasome activation and diet-induced adipose tissue inflammation. J Leukoc Biol 96(6):1087-1100

Ke P, Shao BZ, Xu ZQ, Chen XW, Wei W, Liu C (2017) Activating alpha7 nicotinic acetylcholine receptor inhibits NLRP3 inflammasome through regulation of beta-arrestin-1. CNS Neurosci Ther 23(11):875-884

Kumari N, Kulkarni AA, Lin X, McLean C, Ammosova T, Ivanov A, Hipolito M, Nekhai S, Nwulia E (2015) Inhibition of HIV-1 by curcumin A, a novel curcumin analog. Drug Des Dev Ther 9:5051

Lamkanfi M, Mueller JL, Vitari AC, Misaghi S, Fedorova A, Deshayes K, Lee WP, Hoffman HM, Dixit VM (2009) Glyburide inhibits the Cryopyrin/Nalp3 inflammasome. J Cell Biol 187(1):61-70

Lema C, Varela-Ramirez A, Aguilera RJ (2011) Differential nuclear staining assay for high-throughput screening to identify cytotoxic compounds. Curr Cell Biochem 1(1):1-14

Mares CA, Ojeda SS, Morris EG, Li Q, Teale JM (2008) Initial delay in the immune response to Francisella tularensis is followed by hypercytokinemia characteristic of severe sepsis and correlating with upregulation and release of damage-associated molecular patterns. Infect Immun 76(7):3001-3010

Mariathasan S, Weiss DS, Dixit VM, Monack DM (2005) Innate immunity against Francisella tularensis is dependent on the ASC/ caspase-1 axis. J Exp Med 202(8):1043-1049

Martinon F, Burns K, Tschopp J (2002) The inflammasome: a molecular platform triggering activation of inflammatory caspases and processing of proIL- $\beta$. Mol Cell 10(2):417-426

Periasamy S, Le HT, Duffy EB, Chin H, Harton JA (2016) Inflammasome-Independent NLRP3 restriction of a protective early neutrophil response to pulmonary tularemia. PLOS Pathog 12(12):e1006059
Petrilli V, Dostert C, Muruve DA, Tschopp J (2007) The inflammasome: a danger sensing complex triggering innate immunity. Curr Opin Immunol 19(6):615-622

Pro S (2018) Wavefunction Inc. Irvine, CA, USA

Rathinam VAK, Fitzgerald KA (2016) Inflammasome complexes: emerging mechanisms and effector functions. Cell 165 (4):792-800

Robinson TP, Ehlers T, Hubbard Iv RB, Bai X, Arbiser JL, Goldsmith DJ, Bowen JP (2003) Design, synthesis, and biological evaluation of angiogenesis inhibitors: aromatic enone and dienone analogues of curcumin. Bioorg Med Chem Lett 13(1):115-117

Robinson TP, Hubbard IV RB, Ehlers TJ, Arbiser JL, Goldsmith DJ, Bowen JP (2005) Synthesis and biological evaluation of aromatic enones related to curcumin. Bioorg Med Chem 13 (12):4007-4013

Sharma J, Mares CA, Li Q, Morris EG, Teale JM (2011) Features of sepsis caused by pulmonary infection with Francisella tularensis Type A strain. Microb Pathog 51(1-2):39-47

Thornberry NA, Bull HG, Calaycay JR, Chapman KT, Howard AD, Kostura MJ, Miller DK, Molineaux SM, Weidner JR, Aunins J, Elliston KO, Ayala JM, Casano FJ, Chin J, Ding GJF, Egger LA, Gaffney EP, Limjuco G, Palyha OC, Raju SM, Rolando AM, Salley JP, Yamin T-T, Lee TD, Shively JE, MacCross M, Mumford RA, Schmidt JA, Tocci MJ (1992) A novel heterodimeric cysteine protease is required for interleukin-1[beta]processing in monocytes. Nature 356(6372):768-774

Tisoncik JR, Korth MJ, Simmons CP, Farrar J, Martin TR, Katze MG (2012) Into the eye of the cytokine storm. Microbiol Mol Biol Rev 76(1):16-32

Weiss DS, Henry T, Monack DM (2007) Francisella tularensis: activation of the inflammasome. Ann NY Acad Sci 1105:219-237

Xu Q, Kulkarni AA, Sajith AM, Hussein D, Brown D, Güner OF, Reddy MD, Watkins EB, Lassegue B, Griendling KK (2018) Design, synthesis, and biological evaluation of inhibitors of the NADPH oxidase, Nox4. Bioorg Med Chem 26(5):989-998

Yin H, Guo Q, Li X, Tang T, Li C, Wang H, Sun Y, Feng Q, Ma C, Gao C (2018) Curcumin suppresses IL-1 $\beta$ secretion and prevents inflammation through inhibition of the NLRP3 inflammasome. $\mathrm{J}$ Immunol 200(8):2835-2846 